Marquette University

e-Publications@Marquette

Biological Sciences Faculty Research and

Publications

Biological Sciences, Department of

8-1-2014

\title{
Living Close to Your Neighbors: The Importance of Both Competition and Facilitation in Plant Communities
}

\author{
Alexandra Wright \\ German Centre for Integrative Biodiversity Research \\ Stefan A. Schnitzer \\ Marquette University, stefan.schnitzer@marquette.edu \\ Peter B. Reich \\ University of Minnesota
}

Follow this and additional works at: https://epublications.marquette.edu/bio_fac

Part of the Biology Commons

\section{Recommended Citation}

Wright, Alexandra; Schnitzer, Stefan A.; and Reich, Peter B., "Living Close to Your Neighbors: The Importance of Both Competition and Facilitation in Plant Communities" (2014). Biological Sciences Faculty Research and Publications. 682.

https://epublications.marquette.edu/bio_fac/682 


\title{
Living close to your neighbors: the importance of both competition and facilitation in plant communities
}

\author{
Alexandra Wright, ${ }^{1,2,5}$ Stefan A. Schnitzer, ${ }^{2}$ and Peter B. Reich ${ }^{3,4}$ \\ ${ }^{1}$ German Centre for Integrative Biodiversity Research (iDiv), Halle-Jena-Leipzig, Deutscher Platz 5e, 04103 Leipzig, Germany \\ ${ }^{2}$ Department of Biological Sciences, University of Wisconsin-Milwaukee, P.O. Box 413, Milwaukee, Wisconsin 53201 USA \\ ${ }^{3}$ Department of Forest Resources, University of Minnesota, St. Paul, Minnesota 55108 USA \\ ${ }^{4}$ Hawkesbury Institute for the Environment, University of Western Sydney, Penrith, New South Wales 2751 Australia
}

\begin{abstract}
Recent work has demonstrated that competition and facilitation likely operate jointly in plant communities, but teasing out the relative role of each has proven difficult. Here we address how competition and facilitation vary with seasonal fluctuations in environmental conditions, and how the effects of these fluctuations change with plant ontogeny. We planted three sizes of pine seedlings (Pinus strobus) into an herbaceous diversity experiment and measured pine growth every two weeks for two growing seasons. Both competition and facilitation occurred at different times of year between pines and their neighbors. Facilitation was important for the smallest pines when environmental conditions were severe. This effect decreased as pines got larger. Competition was stronger than facilitation overall and outweighed facilitative effects at annual time scales. Our data suggest that both competition and the counter-directional effects of facilitation may be more common and more intense than previously considered.
\end{abstract}

Key words: diversity; environmental severity; net effects; ontogeny; Pinus strobus; stress gradient hypothesis; theoretical ecology.

\section{INTRODUCTION}

More than 50 years of ecological research have demonstrated that organisms compete for limiting resources in nearly every ecosystem (Hardin 1960, Connell 1961, Ricklefs 1977, Tilman 1977, Brokaw and Busing 2000, Coomes and Grubb 2000). In plant communities, neighboring plants utilize and reduce resources, which ultimately leads to decreased performance at the individual plant level. When competition is strong, experimental removals of neighbor biomass result in increased growth and survival of intact individuals (Casper and Jackson 1997, Schnitzer and Carson 2010). Resource competition is a major determinant of species composition and community dynamics. For example, in North American temperate grasslands, competition for nitrogen and soil water can help explain local successional trajectories (Tilman 1985), the positive relationship between biodiversity and productivity (Isbell et al. 2011), and woody encroachment patterns (Archer et al. 1995, Davis et al. 1999). Competition may be ubiquitous (Tilman 1977), but overwhelming interest in competition experiments may have obscured the co-occurring importance of positive interactions in plant communities (Bertness and Callaway 1994).

Manuscript received 1 October 2013; revised 2 January 2014; accepted 16 January 2014; final version received 7 February 2014. Corresponding Editor: P. B. Adler.

${ }^{5}$ E-mail: sashajwright@gmail.com
Positive interactions (facilitation) among neighbors can promote increased plant growth and survival due to protection from environmental extremes (abiotic facilitation). Specifically, plant communities can reduce direct irradiance, reduce surface soil drying, reduce air and soil temperatures, increase relative humidity, and decrease vapor pressure deficit in their local microclimate (Holmgren et al. 1997, Classen et al. 2010, Montgomery et al. 2010). These direct effects of plant communities on the local microclimate (microclimate amelioration) can, at times, translate to increased performance of resident plants (facilitation). Facilitation is most common in severe environments (the stress gradient hypothesis), where plants experience high levels of physiological stress, and therefore benefit more from the microclimate amelioration effect (Callaway and Pennings 2000, Van Auken 2000, Brooker et al. 2008, Valladares et al. 2008). Indeed, experimental removals of plant biomass in severe environments can result in decreased germination success, survival, and physiological performance (Valladares et al. 2008, Classen et al. 2010, Montgomery et al. 2010), the exact opposite of what is predicted if competition predominates. Theoretically, we may expect these same shifts to happen within single plant communities as environmental conditions change from day to day and season to season.

Plant ontogeny may also influence the relative strengths of competition and facilitation within plant communities (Miriti 2006). During early ontogenetic stages, seedlings may be more vulnerable to abiotic 
stress, and therefore facilitation from neighboring plants may be particularly important (Miriti 2006). Smaller seedlings have relatively less access to deep soil water reserves, and less carbon available in storage organs, than larger plants (Niinemets 2010), and thus are more vulnerable to environmental stressors (Cavender-Bares and Bazzaz 2000). Conversely, larger plants have deeper root systems and more carbon available in storage organs, and are thus more capable of surviving periods of environmental stress. Larger plants, however, also need greater quantities of resources to maintain basal metabolism, which may lead to increased resource use as they grow larger. Therefore, the relative impact of facilitation may decrease with plant ontogeny, while competition intensity may increase.

Empirical studies on facilitation have typically focused on severe environments (tundra, deserts, salt marshes). However, more recent work indicates that facilitation may be more common than originally suggested by the stress gradient hypothesis (Dickie et al. 2005, Montgomery et al. 2010, Wright et al. 2013). Both competition and facilitation may be operating simultaneously in nearly all plant communities, but each process may obscure the relative strength of the other. Thus, the outcome of plant interactions would be the sum of both resource competition, and facilitation due to microclimate amelioration (Bruno et al. 2003). If one of the processes is even slightly stronger than the other, over the course of a single study, the weaker process is typically overlooked and tacitly assumed to be absent. This assumption may be a serious oversight in the interpretation of plant interaction experiments. For example, a positive effect of a neighbor removal on individual plant performance may be due to competitive release. However, neighbor removal may also increase physiological stress, due to the loss of protection from environmental extremes provided by the neighboring canopy. If release from competition has the strongest effect on overall growth (relative to growth lost during periods of physiological strain following neighbor removal), then competitive release will obscure the effect of facilitation, even if the facilitative effect is strong.

We tested the overarching hypothesis that both competition and facilitation are operating in plant communities. However, due to increases in facilitation in severe environments and when plants are small, we predict that the competition/facilitation balance varies with seasonal fluctuations in environmental conditions and plant ontogeny. Though predicted by theory (Bruno et al. 2003), no prior study has identified temporal flips in competition and facilitation between the same neighbors during a single growing season.

We planted three sizes of pine seedlings into an experimental herbaceous plant diversity gradient in central Minnesota, USA. Recent work has identified a diversity gradient as useful for these purposes because it provides a gradient of both types of neighbor interactions; i.e., greater diversity equates to more potential for competition (Tilman et al. 2001) and facilitation (Wright et al. 2013), as both resource supply and microenvironment are altered by increasing plant diversity (Reich et al. 2001, 2012, Adair et al. 2009, Wright et al. 2013). The biotic interactions associated with herbaceous diversity result in part from greater biomass in diverse plots, but additionally from the impacts of greater functional diversity on resources and the microenvironment, as well as increased niche complementarity. Further, the unique manipulation of a diversity gradient brings to the forefront the complexity of biotic and abiotic interactions that are involved between heterospecific and conspecific neighbors in plants communities, as well as the role of diversity in determining the competition/facilitation balance in natural communities.

We measured pine growth at two-week time intervals over the course of two growing seasons. We also measured soil nitrate availability and vapor pressure deficit of the microclimate. We used the increasing strength of competition and facilitation with increasing herbaceous diversity to test three specific hypotheses.

1) The competition-facilitation balance can shift over the course of a season. This is due mostly to fluctuations in environmental conditions.

2) Competition increases with target plant ontogeny (seedling size).

3) Facilitation decreases with target plant ontogeny (seedling size).

\section{Methods \\ Study site and experimental design}

We conducted this study at the Cedar Creek Ecosystem Science Reserve in central Minnesota, USA. Soils at this site consist of nutrient-poor glacial outwash sand plain with low water- and nutrient-holding capacity (Tilman and Wedin 1991). For the purposes of our experiment we utilized ambient treatment plots in the plant diversity gradient in the ongoing BioCON experiment at this site (see Plate 1; Reich et al. 2012). The BioCON plots were established in 1997 by tilling and fumigating existing vegetation in six experimental blocks in an old field. Plots were then seeded with herbaceous species that were selected randomly from a pool of 16 total species from four functional groups (four $\mathrm{C}_{3}$ grasses, four $\mathrm{C}_{4}$ grasses, four legumes, and four non-nitrogen-fixing herbaceous plants). Seeds were divided equally among the species assigned to each plot and applied at a rate of $12 \mathrm{~g} / \mathrm{m}^{2}$ of total seed. Since 1997 , species mixes were maintained using hand weeding to remove any species that migrated into the plot that were not planted in the original seed mix (though plots were not reseeded). Additionally, there were three plots maintained with no vascular plants, wherein all colonizing species were removed (hereafter bareground plots). In total, there were 3 plots with 0 species, 32 
plots with 1 species (with every monoculture represented twice), 32 plots with 4 species, 9 plots with 9 species, and 12 plots with 16 species (88 plots total).

In experimental manipulations of plant diversity, there is often a positive relationship between diversity and biomass production; higher-diversity plots are more productive due to increased diversity of functional types and niche complementarity (Reich et al. 2001, Tilman et al. 2001, Van Ruijven and Berendse 2003, Roscher et al. 2005, Isbell et al. 2011, Reich et al. 2012, Zhang et al. 2012). Consequently, increased diversity in BioCON results in greater competition for resources and greater potential for facilitation under a higher-density canopy (Adair et al. 2011, Reich et al. 2012, Mueller et al. 2013, Wright et al. 2013). For the purposes of this experiment, we utilized this biodiversity gradient to simultaneously manipulate both competition (via reduction in resource availability) and facilitation (via amelioration of environmental conditions). The strength and importance of these relationships were also assessed over the course of our study by measuring nitrate availability and microclimate conditions in our experimental plots.

In June and August of 2010 and 2011 we measured available soil nitrogen (in the form of nitrate) in each of the 88 plots by collecting four soil cores at $0-20 \mathrm{~cm}$ depth, extracting nitrogen using $1 \mathrm{~mol} / \mathrm{L} \mathrm{KCl}$, and analyzing the nitrogen content using a Costech 4050 Element Analyzer (Costech Analytical Technologies, Valencia, California, USA). We also sampled herbaceous biomass each June and August in 2010-2011 in each plot. Aboveground herbaceous biomass was clipped in $10 \times 100 \mathrm{~cm}$ strips at the soil surface, sampled in a different part of the plot each time, and to avoid edge effects, samples were located at least $15 \mathrm{~cm}$ from plot boundaries. Belowground biomass (0-20 $\mathrm{cm}$ ) was obtained using three $5-\mathrm{cm}$ root cores in the same area as the aboveground biomass harvest. We measured air temperature and relative humidity continuously at five-minute intervals (and calculated vapor pressure deficit [VPD]) from May to October 2011 using Maxim iButton dataloggers (Maxim Integrated, San Jose, California, USA). We installed the iButton dataloggers on wooden tent stakes $20 \mathrm{~cm}$ above the ground surface and covered with white plastic cups, which allowed us to accurately measure temperature and relative humidity while reflecting direct sunlight and guarding against direct saturation by rainwater. We installed dataloggers in a stratified subset of 55 plots, which included all monocultures represented once and at least three plots from each of the other species richness levels (assigned randomly within species richness level). We moved dataloggers every month among the plots to capture microclimate conditions across a broader range of species combinations. We measured shallow soil moisture $(0-6 \mathrm{~cm})$ using an $\mathrm{HH} 2$ soil moisture meter (Dynamax, Hous- ton, Texas, USA) every two weeks from May 2010 to October 2010 and May 2011 to October 2011.

\section{Seedling growth}

In June 2010 we planted 11 white pine (Pinus strobus) seedlings into each of the plots. Plants were germinated and initially reared by Vans Pines Nursery (West Olive, Michigan, USA) from Michigan pine seed. Seedlings were grown as bare-root stock for the first year and then grown in nursery soil until they were shipped to us. Before planting, all roots were rinsed of nursery soil. All seedlings were 1-3 years old at time of planting. In each plot we planted three large-size seedlings $(>15 \mathrm{~cm}$ tall), three medium-size seedlings $(10-15 \mathrm{~cm}$ tall), and five small-size seedlings $(<5 \mathrm{~cm}$ tall $)$, as we predicted that survival rates of the smallest size class would be the lowest (Wright et al. 2013). We measured pine basal diameter and height every two weeks from 16 June 2010 to 19 October 2010, and again from 10 May 2011 to 22 September 2011, at which point all pines were harvested. We harvested pine aboveground biomass (AGB) by clipping pine stems at the soil surface, but belowground biomass was not collected for the sake of the long-term integrity of the BioCON experimental plots.

To estimate the relationship between biweekly size measurements (i.e., every two weeks) taken in the field (height and basal diameter) and plant biomass, we planted 10 large, 10 medium, and 20 small pine seedlings in a harvestable plot near the BioCON experiment. We harvested these seedlings throughout the first growing season and preserved the aboveground and belowground biomass components of all individuals. We dried and weighed all biomass from this final pine harvest $(n=255)$ and from the harvest garden $(n=40)$. We pooled all samples (from the harvest plot and the BioCON final harvest) to calculate aboveground biomass at every census from field measurements, and used the harvest plot data to estimate belowground biomass (Appendix A: Table A1). Based on differences in tree allometry at small sizes (Mascaro et al. 2011), and the fact that a single allometry equation overestimated biomass predictions for the smallest size class, we fit a separate allometric relationship for the small size class, and one combined allometric relationship for the large and medium size classes $\left(r^{2}>0.74\right.$ for all allometric relationships [Appendix A: Table A1]). We calculated relative growth rate (RGR) using the equation: [n(total final biomass) - $\ln$ (total initial biomass)]/time interval (Poorter and Lewis 1986).

\section{Analyses}

To assess the relationship between diversity, resource availability, and microclimate, over the course of our study, we analyzed the effects of herbaceous diversity on microclimate factors (shallow soil moisture, air temperature, relative humidity, and vapor pressure deficit) and soil nitrogen, using a mixed-effects ANCOVA for 
repeated measures. Within the mixed-effects framework, the BioCON experimental block ("ring") was included as a random effect. Species richness was considered a continuous fixed effect, and the environmental factors were included as continuous response variables. Time (year or biweekly interval) was included as a random effect to account for repeated measurements taken on the same plots over time.

To determine whether pine RGR varied with seedling size and census interval across the diversity gradient, we used a mixed-effects ANCOVA as described above ("ring" is a random effect). For these analyses, multiple pine seedlings were planted within each plot, so plot number was nested within "ring" to account for lack of independence among seedlings within a single plot. To determine whether the biweekly measurements of competition and facilitation differed from the net combined effects of competition and facilitation over the two years of our study we analyzed our data set at two time scales:

1. Coarse temporal scale.-To test whether plant competition or facilitation was the dominant process operating on each seedling at longer time intervals, we measured growth each year (RGR for 4 June 2010-10 October 2010 and 1 June 2011-26 September 2011). We used the annual time scale (instead of a single point at the beginning and end of the study) because most studies measure growth annually. Also, this approach allowed us to include individuals that may have died before the end of the study, but were alive and growing in the first year. Further, to assess the overall effects of diversity and seedling size on seedling growth, without focusing on interannual variation, we included the main effects of pine seedling size and herbaceous diversity as fixed effects in our model, and included year in the model as a random effect to account for autocorrelation of measurements taken on the same plant over time (further details in Appendix B: Eq. B.1).

2. Fine temporal scale.-To determine the underlying variation in competition and facilitation over short time intervals, we used biweekly measurements of all individuals over the course of two growing seasons. For this analysis, biweekly census interval was included in the statistical model as a fixed effect. The inclusion of census interval allowed us to directly analyze whether the effects of diversity and size class changed from week to week, and whether competition and facilitation change depending on seasonal changes in environmental severity. To account for autocorrelation of measurements taken on the same plant over time, plant identity was nested in the random effects term described previously. This was done using a mixed-effects model for repeated measures design (Pinheiro and Bates 2000; further details in Appendix B: Eq. B.2).

Soil nitrate and microclimate as explanatory mechanisms for pine seedling growth.-We conducted separate analyses to assess how soil nitrate and microclimate directly affected seedling growth, and how these mechanisms for competition and facilitation changed with seedling size. Because soil nitrate was only measured twice per year and averaged annually, we assessed the effects of nitrate, size class, and interactions using a mixed-effects ANCOVA at the coarse temporal scale (in the same model framework as above). We assessed the effects of VPD, soil moisture, size class, and interactions, using a mixed-effects ANCOVA at the fine temporal scale (in the same model framework as previously).

To understand how pine growth was affected by between-plot variation in biomass production that occurred within species richness levels (e.g., if observed effects are more directly related to biomass effects), we also added total herbaceous biomass to the mixedeffects ANCOVA and analyzed these effects at the coarse temporal scale. To do this we averaged the June and August herbaceous biomass harvest data to obtain an annual measurement for each plot in each year.

\section{Results}

\section{Net effects and size-structured effects}

Over the two years (coarse temporal scale), pines growing in higher-diversity plots grew less than pines growing in lower-diversity plots (species richness main effect, $\left.F_{1,87}=29.3, P<0.0001\right)$. In other words, the net effect of diversity on pine growth was negative, indicating that competition increased more rapidly with increasing diversity than facilitation did. Smaller seedlings had a lower RGR overall than larger seedlings, but the growth of larger seedlings was more negatively affected by diversity (species richness $\times$ size interaction, $F_{2,605}=8.24, P=0.0003$; Appendix C: Table C1). Specifically, small seedlings grew equally well across diversity treatments (Fig. 1a), whereas medium and large seedlings were limited by diversity (Fig. 1b, c), with large seedlings showing the strongest response (Fig. 1c). These effects were not strongly influenced by variation in herbaceous biomass production (Appendix C: Table C1).

\section{Soil nitrate, shallow soil water, and microclimate}

Higher-diversity plant communities (16 species) had half the soil nitrate of bareground plots $\left(F_{1,86}=6.27, P=\right.$ $0.01)$. Long-term averages of shallow soil moisture and relative humidity both increased with diversity, whereas air temperature and vapor pressure deficit decreased with diversity (Fig. 2). Shallow soil moisture was on average $1 \%$ greater in 16 -species plots $(\sim 8.5 \%$ soil moisture) than in 0 - and 1 -species plots $(\sim 7.5 \%$ soil moisture, $F_{1,84}=11.3, P=0.001$; Fig. 2a). Mean air temperature was $\sim 2^{\circ} \mathrm{C}$ cooler in 16-species plots than in 1 -species plots $\left(F_{1,50}=24.7, P<0.0001\right.$; Fig. 2b). Average relative humidity was $\sim 12 \%$ higher in 16 species plots (from $77 \%$ relative humidity to $89 \%$ relative humidity, $F_{1,52}=47.1, P<0.0001$; Fig. 2c). Average vapor pressure deficit was reduced by $75 \%$ in 16 -species 


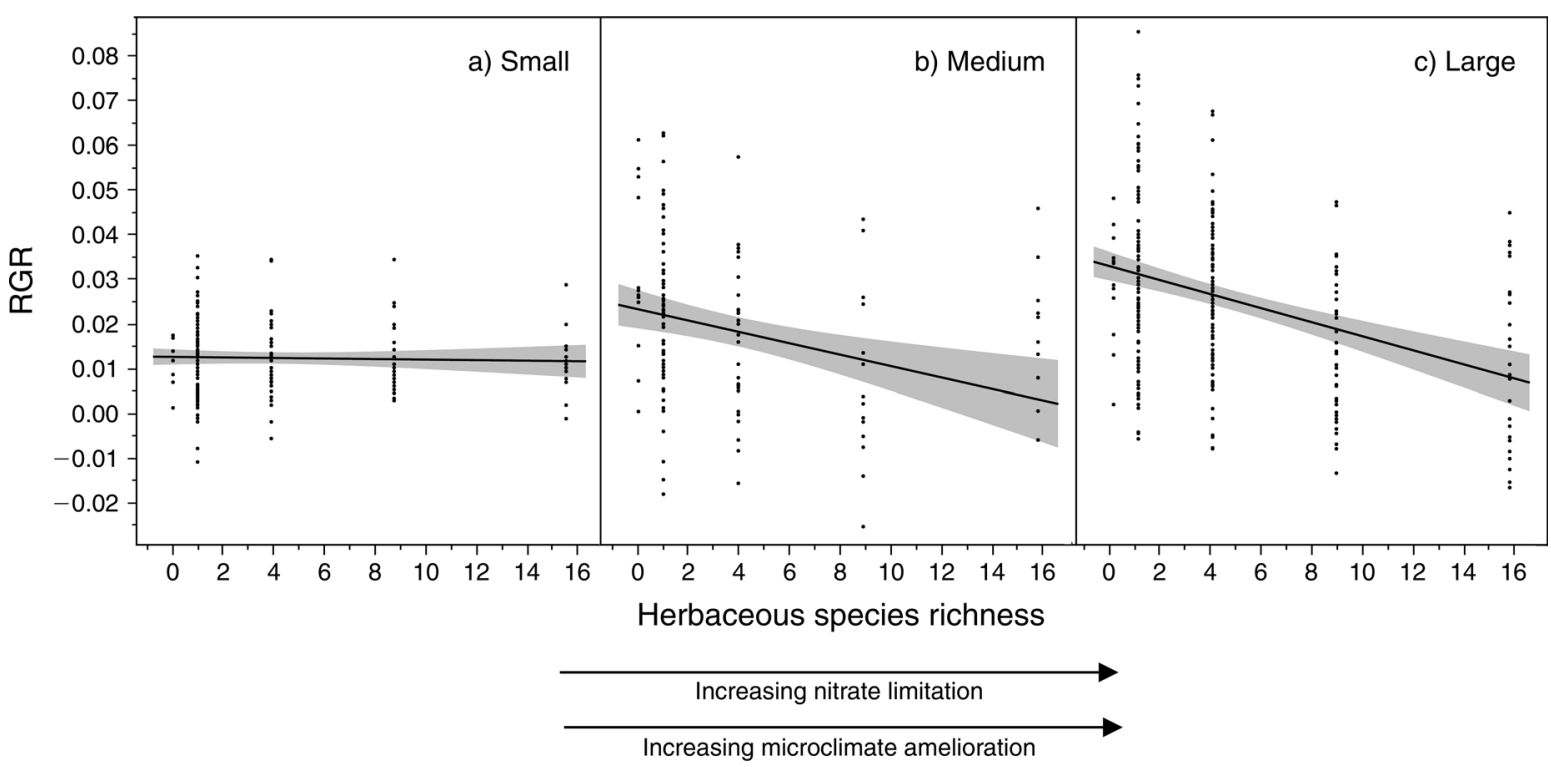

FIG. 1. Coarse-resolution measurements of pine relative growth rate (RGR) for both years. The change in seedling response with ontogenetic stage demonstrates the interaction between species richness and pine size class: larger pines are more negatively affected by species richness. Shading represents $95 \%$ confidence intervals.
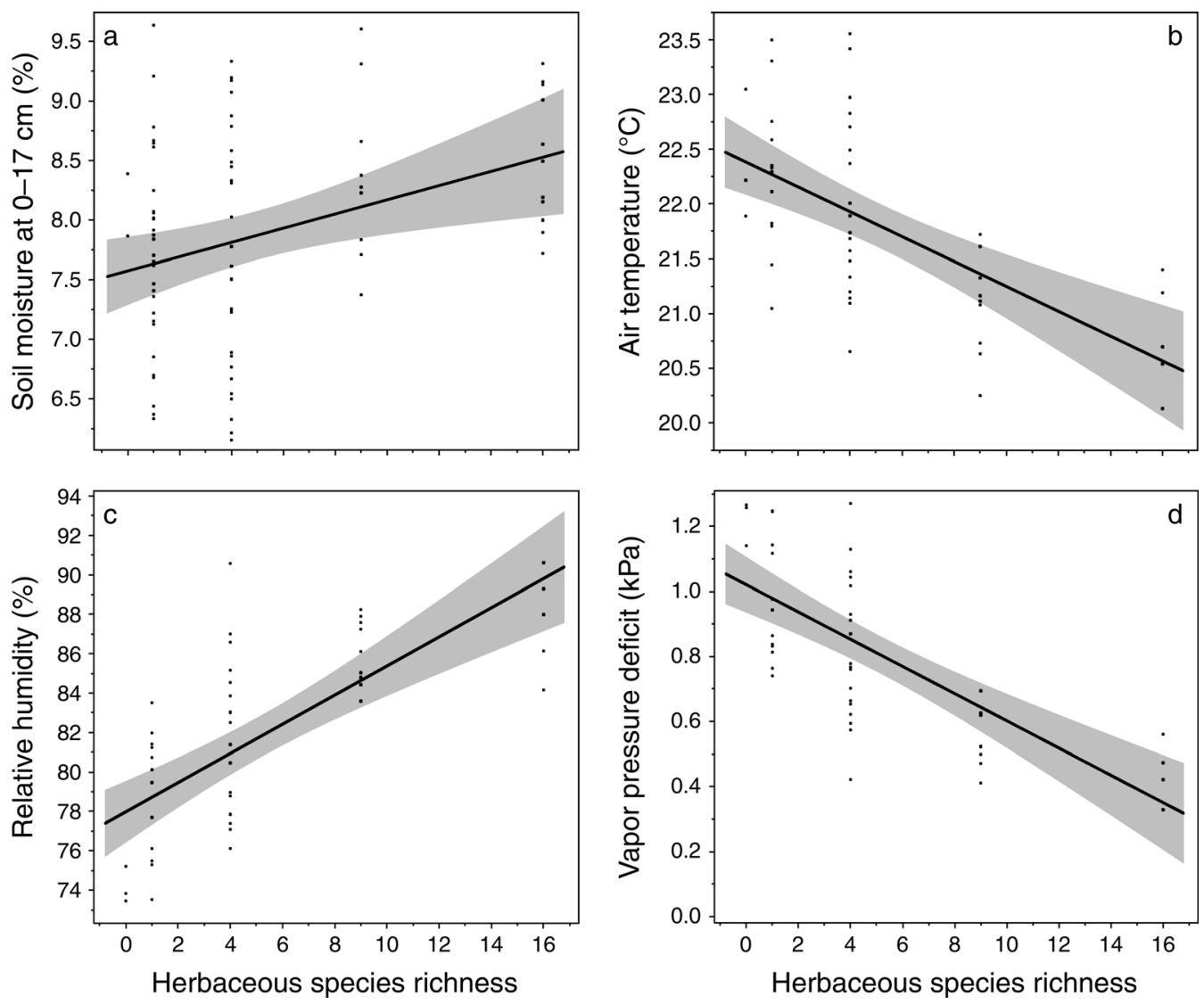

FIG. 2. The effects of increasing herbaceous species richness on microclimate conditions. The panels demonstrate the relationship between richness and (a) shallow soil moisture, (b) temperature, (c) relative humidity, and (d) vapor pressure deficit. Air temperature, relative humidity, and vapor pressure deficit are presented as averages for all 24-hour periods from May to September 2011. Shading represents $95 \%$ confidence intervals. 


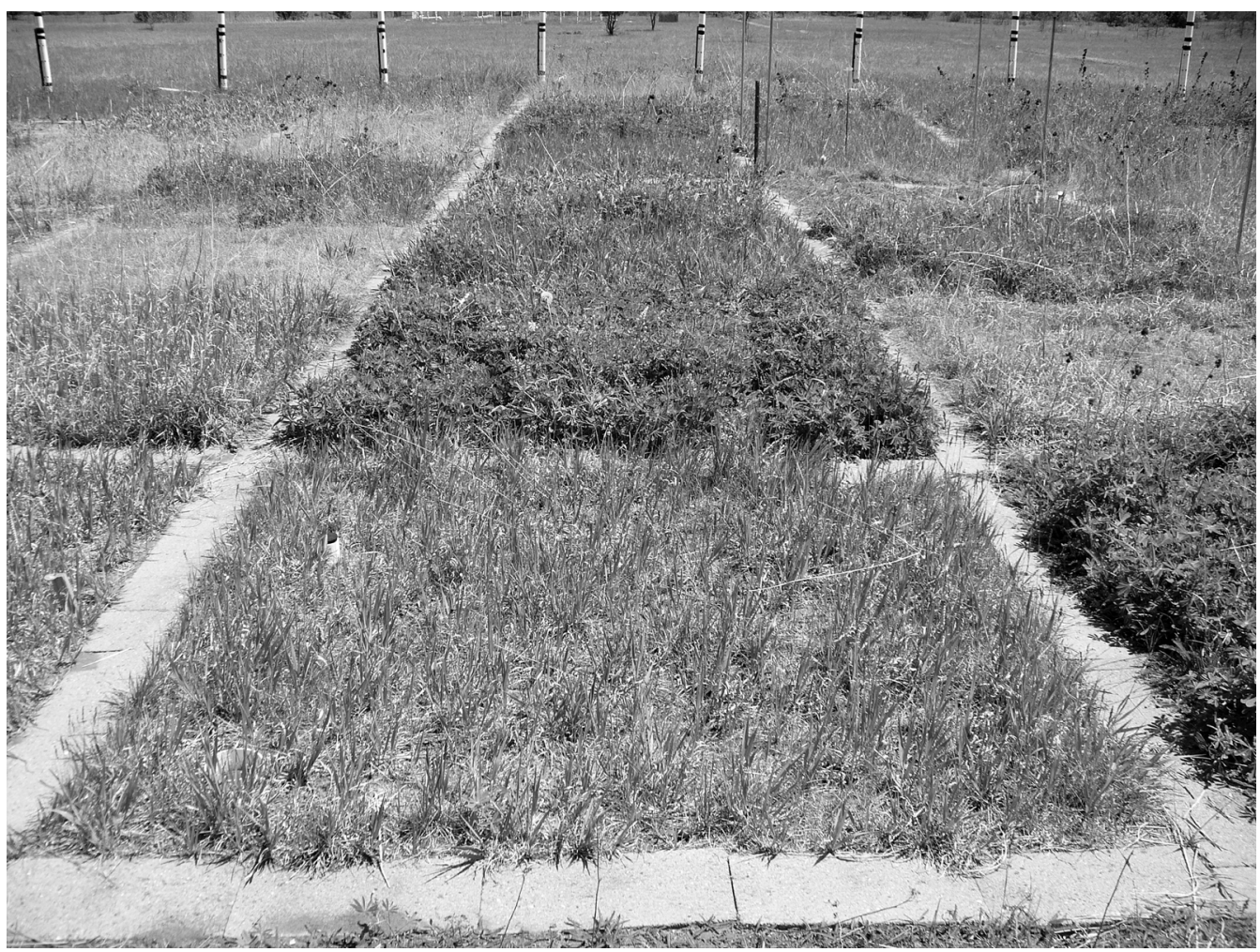

Plate 1. The biodiversity manipulation was conducted using 1,4 , 9, or 16 species planted as seed into $2 \times 2 \mathrm{~m}$ plots (demarcated with cinderblock paths in the image). Photo credit: A. Wright.

plots compared with 1 -species plots $\left(F_{1,52}=55.9, P<\right.$ 0.0001; Fig. 2d).

\section{Relative strengths of competition and facilitation change with seedling size}

Biweekly measurements of pine seedling RGR revealed that competition was a stronger force than facilitation with increasing diversity in the majority of sampling intervals (when averaged over seedling sizes, the species richness main effect was significant and negative, $\left.F_{1,465}=48.2, P<0.0001\right)$. However, whether competition or facilitation was a stronger force changed significantly from one census to the next, and this was particularly true for small individuals $(P<0.0001$ for the three-way interaction; Table 1, Fig. 3). Small seedlings exhibited competitive, neutral, and facilitative responses in different census intervals. Additionally, within a single census interval the effects of diversity could be strongly competitive for large individuals and neutral for small individuals, yet within another interval the effects could be facilitative for small individuals and neutral for larger individuals (Appendix D: Fig. D1).

Seedlings grew more in plots with greater nitrate availability $\left(F_{1,71}=7.66, P=0.007\right)$; this effect did not change with seedling size (no interaction of size $\times$ nitrate availability, $F_{2,363}=1.66, P=0.19$ ). Thus, seedlings of all size classes were equally limited by access to soil nitrate (Fig. 4a). There was no overall effect of shallow soil moisture or vapor pressure deficit (VPD) on seedling RGR $\left(F_{1,263}=0.07, P=0.80\right)$, although there was an interaction between seedling size and the effect of VPD on growth; small-seedling RGR was significantly reduced when vapor pressure deficit was high, whereas

TABLE 1. The effects of herbaceous species richness and seedling size class on seedling relative growth rate for each individual two-week census interval.

\begin{tabular}{lccc}
\hline \hline \multicolumn{1}{c}{ Fixed effect } & $\mathrm{df} \dagger$ & $F$ & $P$ \\
\hline Species richness & 1,465 & 48.2 & $<\mathbf{0 . 0 0 0 1}$ \\
Size class & 2,386 & 59.8 & $<\mathbf{0 . 0 0 0 1}$ \\
Census & 15,5967 & 69.7 & $<\mathbf{0 . 0 0 0 1}$ \\
Species richness $\times$ size & 2,456 & 0.99 & 0.37 \\
Species richness $\times$ census & 15,6015 & 2.53 & $\mathbf{0 . 0 0 1}$ \\
Size $\times$ census & 30,5969 & 25.0 & $<\mathbf{0 . 0 0 0 1}$ \\
Species richness $\times$ size $\times$ census & 30,6022 & 3.97 & $<\mathbf{0 . 0 0 0 1}$ \\
\hline
\end{tabular}

Note: In order to avoid pseudo-replication of measurements taken on the same individuals over time, seedling ID was included as a random effect in the statistical model (as seen in Appendix B: Equation B.2). Significant $P$ values are shown in boldface type.

$\dagger$ This analysis took into account spatial variation associated with the blocked design ("Ring" in the BioCON framework) as well as variation associated with taking measurements on multiple seedlings within one plot ("plot") and on the same individuals over time (seedling ID). This is why denominator degrees of freedom are different depending on the metric described in this table. 

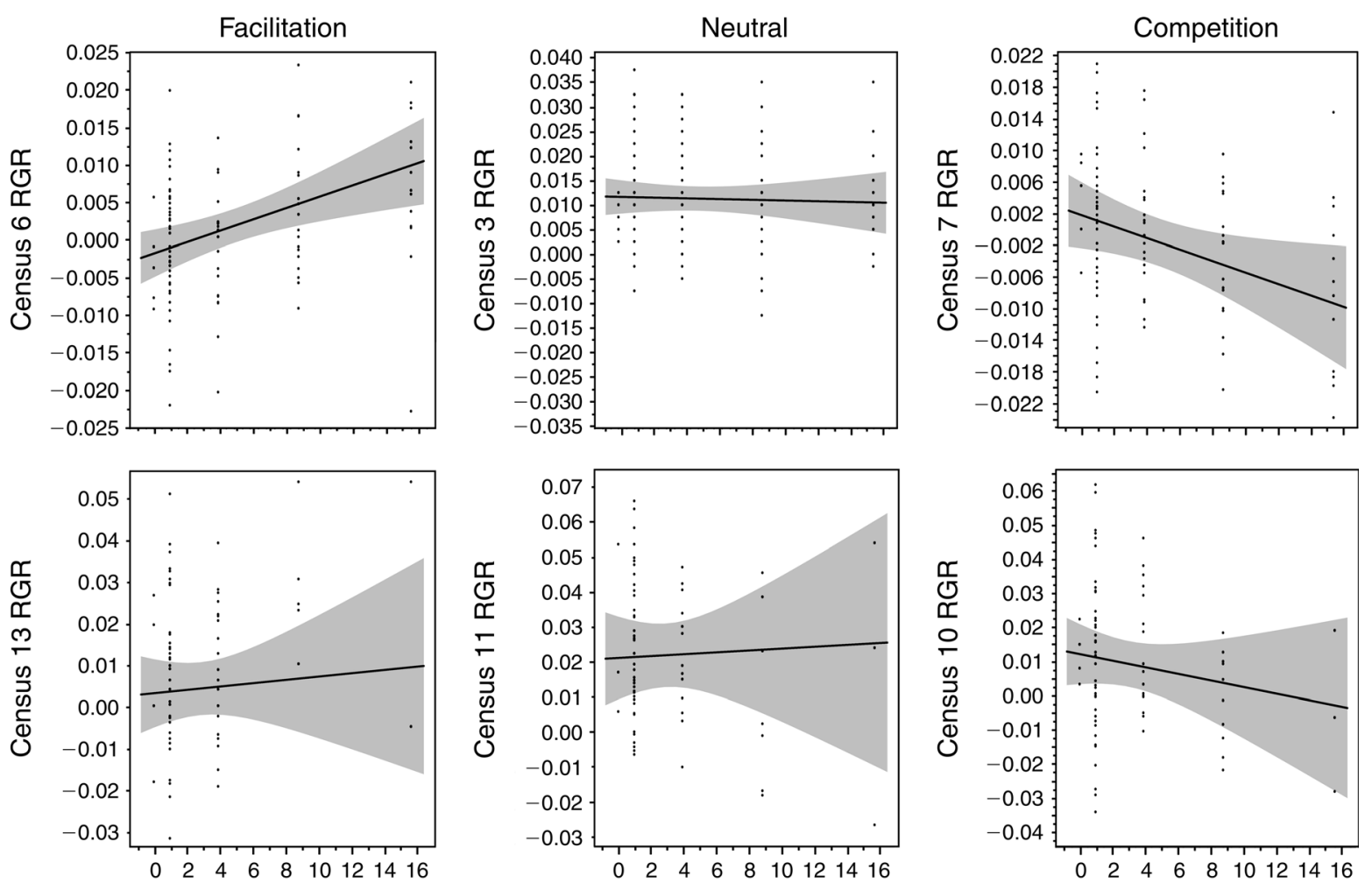

Herbaceous species richness

FIG. 3. Pine relative growth rate (RGR) varied over the course of the study, particularly for the smallest pines. Here we show instances in both years where small pine growth varied from positive (facilitation), to neutral, to negative (competition). Shading represents $95 \%$ confidence intervals.

larger seedlings were not affected by changes in VPD $\left(F_{2,1326}=5.27, P=0.005\right.$; Fig. $\left.4 b\right)$.

\section{Discussion}

We found that both competition and facilitation influenced pine seedling growth rates in this experimental grassland community. Overall pine growth was most limited by access to soil nitrate (and likely other limiting resources) in the highest-diversity plots. Thus it would be easy to conclude that competition was the driving force of all observed interactions, while assuming facilitation to be absent. However, by explicitly manipulating plant size, we found that the intensity of this growth limitation increased with seedling size (Fig. 1). Again, a common interpretation of increasing growth limitation with increasing size may be increasing competition intensity experienced by larger individuals (Fig. 5b). However, competition for soil nitrate was not more intense for larger seedlings, meaning that larger seedlings may not have been more limited by competition for this important limiting nutrient. (The competition slope remains the same; Fig. 5a.) A more likely explanation for increasing growth limitation with increasing seedling size, in this case, is a decrease in facilitation intensity as seedlings grow (Fig. 5c). Indeed, smaller seedling growth was facilitated by reduced stress associated with vapor pressure deficit, and this effect decreased with seedling size (Fig. 4b). While the net effect of plant interactions in this system may be competitive, the combined effects of competition and facilitation determined overall plant growth patterns.

Cedar Creek Ecosystem Science Reserve has a continental climate (warm summers and cold winters) and receives $\sim 32 \mathrm{~cm}$ of precipitation on average during summer months (Davis et al. 2005, Peel et al. 2007). This is sufficient rainfall to be classified as a humid continental climate as opposed to arid or semiarid (Peel et al. 2007). In terms of plant desiccation stress, this system may not be particularly stressful in comparison to deserts and arctic habitats, but the sandy soil likely leads to periodic water stress during the summer months. Past research at this site has strongly emphasized the importance of competition for community composition, succession, and overall plant diversity in both experimental (Tilman et al. 2001) and observational settings (Tilman and Wedin 1991), including for woody seedlings colonizing herb-dominated patches (Davis et al. 1998, 1999). Annual pine RGR measurements in our experiment reflect similar patterns: when measured at coarse time scales, the only detectable pattern was decreasing pine growth in higher-diversity plots, and this negative growth response was stronger in 

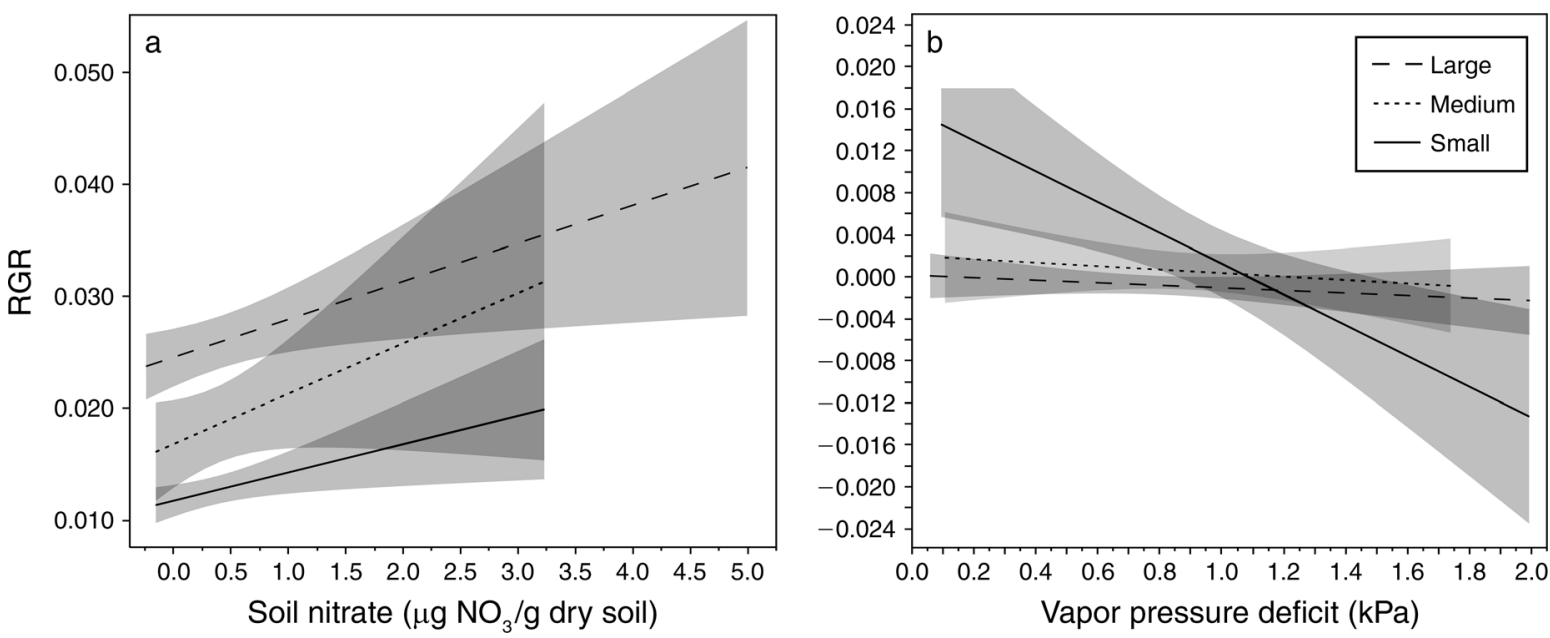

FIG. 4. (a) Relative growth rate of all seedlings is equally limited by access to available soil nitrogen in the form of nitrate. Conversely, (b) there is a significant interaction between vapor pressure deficit and seedling size. Small individuals are strongly negatively affected by high vapor pressure deficit, whereas large and medium individuals are not significantly affected. Shading represents $95 \%$ confidence intervals.
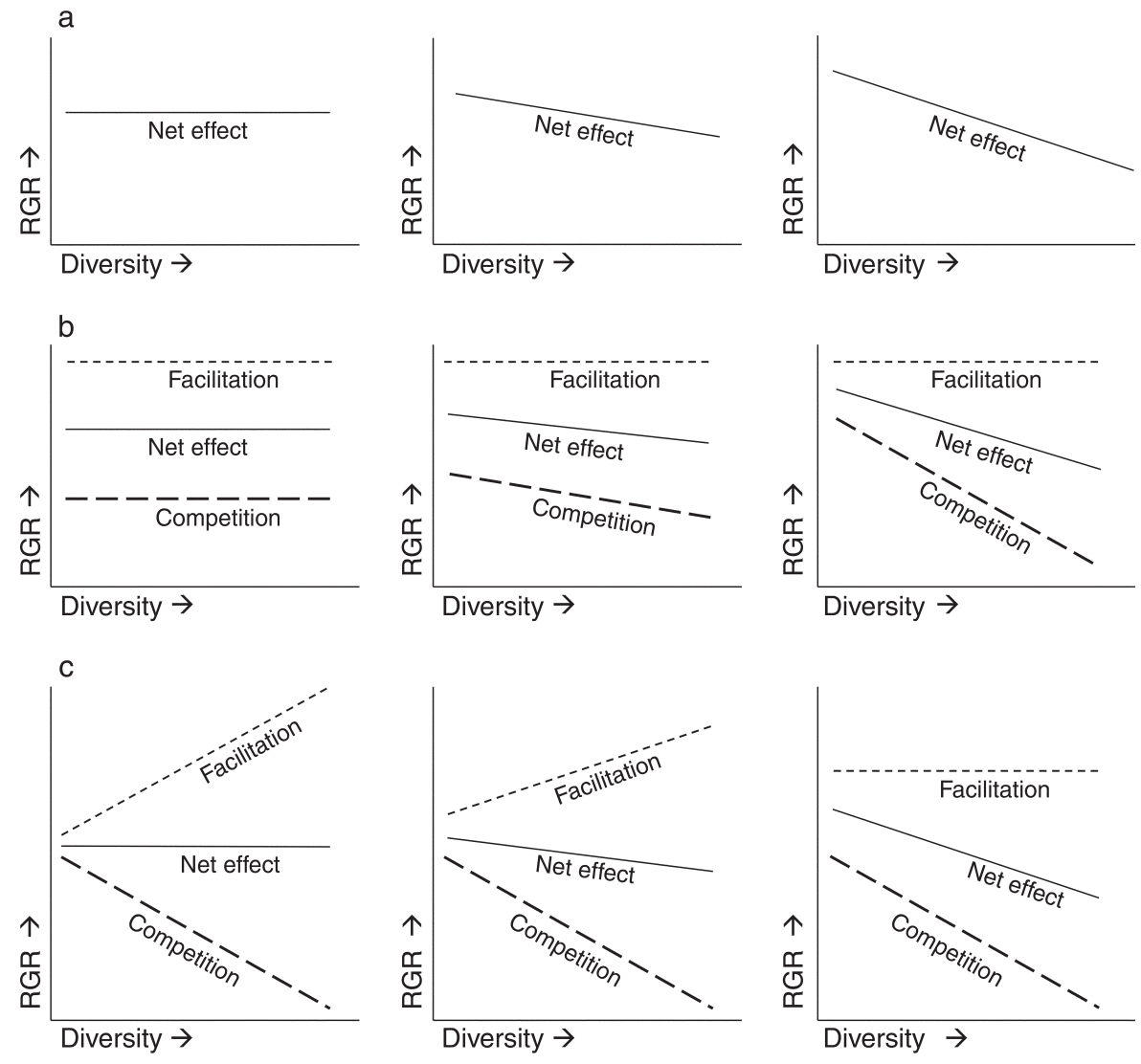

FIG. 5. (a) Conceptual diagram of net effects showing that observed effects of diversity on pine relative growth rate may indicate increasing competition with increasing seedling size. However, underlying competition and facilitation may help explain these observations in several different ways. (b) Competition intensity may increase with increasing seedling size, or (c) facilitation intensity may decrease with increasing seedling size. This diagram is for conceptualization only. The net effects line is displayed as the midpoint between the competition and facilitation lines. 
larger seedlings. Decreased growth is usually interpreted as increased competition intensity at higher levels of herbaceous plant diversity (Fargione and Tilman 2005; but see Schnitzer et al. 2011). However, while competition may be stronger than facilitation at the coarse time scale, the effects of facilitation may profoundly structure seedling growth and neighbor interactions during periods of environmental stress; these periods of stress lead to growth reduction in low not high diversity plots, and these counter-directional effects contribute to overall pine growth patterns. In other words, if it weren't for the effects of facilitation, we may have seen small pines excluded completely in some cases, and large pines experiencing even stronger competitive effects at all diversity levels.

Facilitation is important for small seedlings because they are particularly sensitive to harsh environmental conditions. Small seedlings have reduced access to deep soil water reserves and may be vulnerable during periodic droughts. Small seedlings also have fewer nonstructural carbohydrates available for maintaining plant metabolic activity during brief periods of stress (Niinemets 2010). These physiological and physical constraints make smaller seedlings more vulnerable to environmental stressors, and therefore more dependent on the facilitative microhabitat amelioration provided by neighbors. We found that the microclimate under the canopy of a high-diversity community is cooler, more humid, and has higher soil water content at the soil surface than a lower-diversity community (Fig. 2). Interestingly, previous work has demonstrated that soil moisture at greater soil depths $(>6 \mathrm{~cm})$ is lower in highdiversity communities (Adair et al. 2011), due mostly to greater competition for soil water at depth. Conversely, surface soil moisture seems to be more strongly controlled by evaporation at the soil surface (Wright et al. 2013). Evaporation is higher in lower-diversity plots because a greater proportion of the soil surface is exposed. Our results demonstrate that small seedlings have increased vulnerability to environmental stress (Fig. 4b), and that they can periodically benefit from growing in higher-diversity communities where there is greater amelioration of these conditions (Fig. 2). Mechanistically, this same pattern may be conceptualized as a switch from competition for soil water when plants are small (and have shallower roots) to competition for soil nitrate as plants grow larger. Competition for shallow soil water is more intense in lower-diversity plots (due to lack of facilitation), while competition for deep soil nitrate is more intense in higher-diversity plots.

Facilitation at the seedling establishment phase may also help explain overall colonization rates and the composition of seedlings in their first year of growth. If the first filter for plant community composition relies heavily on protection from environmental severity, we may expect greater overall colonization rates in higherdiversity communities. Further, if adults tend to facilitate heterospecific seedling establishment more then they facilitate first-year conspecifics, this could lead to a positive feedback loop that increases species diversity in the youngest seedling layer, particularly when environmental conditions are severe (Bertness and Callaway 1994, Brooker 2006). Future work should attempt to determine if interspecific facilitation is more common than intraspecific facilitation at any life history stage, particularly for small, colonizing seedlings, as this type of interaction would contribute to our understanding of diversity maintenance mechanisms.

An overwhelming proportion of past work on plant diversity has emphasized the importance of competition for resources with increasing species richness (Reich et al. 2001, Tilman et al. 2001, Van Ruijven and Berendse 2003, Roscher et al. 2005, Isbell et al. 2011, Reich et al. 2012, Zhang et al. 2012). Particularly in experimental manipulations of species richness, an increase in richness is related to decreased resource availability and increased competition intensity. Here we utilize that pattern to implicitly manipulate competition intensity between plants by manipulating species richness of plots. Conversely, a relationship between manipulated plant diversity and facilitation intensity is much less well represented in the literature and less intuitive to most ecologists (but see Wright et al. 2013). Here we show that microclimate amelioration increases sharply with increasing levels of plant diversity. This pattern is presumably due to the species richness manipulation itself; higher-diversity plots are denser and therefore more likely to result in stronger plantplant interactions of all types (e.g., living close to your neighbors results in increased competition and facilitation intensity). However, both the competition and facilitation effects we observed were due only partially to increased biomass production (increased density) in higher-diversity plots.

Past work has demonstrated that a large component of increased competition intensity with increasing diversity in biodiversity experiments is due to niche complementarity and functional diversity, not just differences in biomass. Higher-diversity plots contain more species with unique strategies for accessing limiting resources. Increased diversity, not biomass per se, leads to increased resource consumption (Loreau and Hector 2001, Craine et al. 2003, Reich et al. 2012). Extending this same logic to facilitation, one potential explanation for the diversity-microclimate amelioration effect may be that higher-diversity plots are more likely to include particularly productive species (Tilman and Downing 1994), or particularly drought-resistant species, that are capable of high photosynthetic rates even on hot/dry days. Increased photosynthesis, water loss, and evaporative cooling at the herbaceous leaf surface may increase the microclimate amelioration effect of herbaceous diversity, which may be magnified by a diversity of growth strategies in response to environmental conditions, rather than by biomass alone. A second potential explanation is that complementarity in leaf shape and 
plant architecture may lead to increased light interception in higher-diversity plots that cannot be explained by biomass alone (Loreau and Hector 2001). Both explanations are non-mutually exclusive and may help explain the unique relationship between diversity and microclimate amelioration observed in our study.

Our data support the hypothesis that competition and facilitation are both operating between neighboring plants in plant communities (Bruno et al. 2003, Brooker 2006). When competition is predominant, positive interactions can easily be overlooked, and assumed to be absent. To our knowledge, no prior study has identified temporal shifts in the biotic effects occurring between the same neighbors. These data vividly demonstrate not only that joint competitive and facilitative effects can occur, but that specific neighbor interactions can shift from one to the other during a single growing season. The implications of this are potentially great. In our study system, if the net effect of diversity were merely the result of competition, we may have observed little growth and establishment of the smallest pines and perhaps much stronger competition intensity for the largest pines in the highest-diversity plots. Instead we observed patterns that appear to reflect a combination of competitive effects and counteracting facilitative effects. The outcome is a balance between the two. Furthermore, if the observed neighbor interactions are the result of competition drivers (resource supply and demand) as well as facilitation drivers (environmental severity, temperature, drought) our expectations for community composition and diversity in the future may differ substantially from our current predictions (Brooker 2006). Furthermore, our past and future interpretation of interactions in plant communities may be greatly altered if we integrate the importance of both competition and the counter-directional effects of facilitation into our understanding of how plant communities function.

\section{ACKNOWLEDGMENTS}

This research was supported by the DOE Program for Ecological Research Grant DE-FG02-96ER62291, the National Science Foundation Long-term Ecological Research Grant DEB-0080382, the NSF Long-term Research in Environmental Biology Grant DEB-0716587, the University of Minnesota, the UWM Department of Biological Sciences, and the NSF Graduate Research Fellowship Program. We thank the help of two anonymous reviewers, J. Husney, K. Worm, S. Barrott, J. Strini, H. Kruse, B. Heim, and the intern crew at Cedar Creek for field assistance during the collection of pine growth data. A. Wright collected the data pertaining to pine seedling growth, P. B. Reich collected the main data for BioCON experiment, A. Wright analyzed the data and wrote the first draft of the manuscript, and all authors contributed substantially to revisions.

\section{Literature Cited}

Adair, E. C., P. B. Reich, S. E. Hobbie, and J. M. H. Knops 2009. Interactive effects of time, $\mathrm{CO}_{2}, \mathrm{~N}$, and diversity on total belowground carbon allocation and ecosystem carbon storage in a grassland community. Ecosystems 12:1037-1052.
Adair, E. C., P. B. Reich, J. J. Trost, and S. E. Hobbie. 2011. Elevated $\mathrm{CO}_{2}$ stimulates grassland soil respiration by increasing carbon inputs rather than by enhancing soil moisture. Global Change Biology 17:3546-3563.

Archer, S., D. Schimel, and E. Holland. 1995. Mechanisms of shrubland expansion: land use, climate or $\mathrm{CO}_{2}$ ? Climatic Change 29:91-99.

Bertness, M., and R. Callaway. 1994. Positive interactions in communities. Trends in Ecology and Evolution 9:191-193.

Brokaw, N., and R. Busing. 2000. Niche versus chance and tree diversity in forest gaps. Trends in Ecology and Evolution 15: 183-188.

Brooker, R. 2006. Plant-plant interactions and environmental change. New Phytologist 171:271-284.

Brooker, R. W., et al. 2008. Facilitation in plant communities: the past, the present, and the future. Journal of Ecology 96: $18-34$.

Bruno, J., J. Stachowicz, and M. Bertness. 2003. Inclusion of facilitation into ecological theory. Trends in Ecology and Evolution 18:119-125.

Callaway, R. M., and S. C. Pennings. 2000. Facilitation may buffer competitive effects: indirect and diffuse interactions among salt marsh plants. American Naturalist 156:416-424.

Casper, B. B., and R. B. Jackson. 1997. Plant competition underground. Annual Review of Ecology and Systematics 28: $545-570$.

Cavender-Bares, J., and F. A. Bazzaz. 2000. Changes in drought response strategies with ontogeny in Quercus rubra: implications for scaling from seedlings to mature trees. Oecologia 124:8-18.

Classen, A., R. Norby, C. Campany, and K. Sides. 2010. Climate change alters seedling emergence and establishment in an old-field ecosystem. PLoS ONE 5:e13476.

Connell, J. 1961. The influence of interspecific competition and other factors on the distribution of the barnacle Chthamalus stellatus. Ecology 42:710-723.

Coomes, D. A., and P. J. Grubb. 2000. Impacts of root competition in forests and woodlands: a theoretical framework and review of experiments. Ecological Monographs 70: 171-207.

Craine, J., P. Reich, G. David Tilman, D. Ellsworth, J. Fargione, J. Knops, and S. Naeem. 2003. The role of plant species in biomass production and response to elevated $\mathrm{CO}_{2}$ and N. Ecology Letters 6:623-625.

Davis, M., L. Bier, E. Bushelle, C. Diegel, A. Johnson, and B. Kujala. 2005. Non-indigenous grasses impede woody succession. Plant Ecology 178:249-264.

Davis, M., K. Wrage, and P. Reich. 1998. Competition between tree seedlings and herbaceous vegetation: support for a theory of resource supply and demand. Journal of Ecology 86:652-661.

Davis, M., K. Wrage, P. Reich, M. Tjoelker, T. Schaeffer, and C. Muermann. 1999. Survival, growth, and photosynthesis of tree seedlings competing with herbaceous vegetation along a water-light-nitrogen gradient. Plant Ecology 145:341-350.

Dickie, I. A., S. A. Schnitzer, P. B. Reich, and S. E. Hobbie. 2005. Spatially disjunct effects of co-occurring competition and facilitation. Ecology Letters 8:1191-1200.

Fargione, J. E., and D. Tilman. 2005. Diversity decreases invasion via both sampling and complementarity effects. Ecology Letters 8:604-611.

Hardin, G. 1960. The competitive exclusion principle. Science 131:1292-1297.

Holmgren, M., M. Scheffer, and M. Huston. 1997. The interplay of facilitation and competition in plant communities. Ecology 78:1966-1975.

Isbell, F., et al. 2011. High plant diversity is needed to maintain ecosystem services. Nature 477:199-202.

Loreau, M., and A. Hector. 2001. Partitioning selection and complementarity in biodiversity experiments. Nature 412:7276. 
Mascaro, J., C. M. Litton, R. F. Hughes, A. Uowolo, and S. A. Schnitzer. 2011. Minimizing bias in biomass allometry: model selection and log-transformation of data. Biotropica 43:649653.

Miriti, M. N. 2006. Ontogenetic shift from facilitation to competition in a desert shrub. Journal of Ecology 94:973979.

Montgomery, R. A., P. B. Reich, and B. J. Palik. 2010. Untangling positive and negative biotic interactions: views from above and below ground in a forest ecosystem. Ecology 91:3641-3655.

Mueller, K. E., D. Tilman, D. A. Fornara, and S. E. Hobbie. 2013. Root depth distribution and the diversity-productivity relationship in a long-term grassland experiment. Ecology 94: 787-793.

Niinemets, Ã. 2010. Responses of forest trees to single and multiple environmental stresses from seedlings to mature plants: past stress history, stress interactions, tolerance and acclimation. Forest Ecology and Management 260:16231639.

Peel, M. C., B. L. Finlayson, and T. A. McMahon. 2007. Updated world map of the Köppen-Geiger climate classification. Hydrology and Earth System Sciences Discussions 4: 439-473.

Pinheiro, J. C., and D. Bates. 2000. Mixed-effects models in S and S-PLUS. Springer, New York, New York, USA.

Poorter, H., and C. Lewis. 1986. Testing differences in relative growth rate: a method avoiding curve fitting and pairing. Physiologia Plantarum 67:223-226.

Reich, P., J. Knops, D. Tilman, J. Craine, D. Ellsworth, M. Tjoelker, T. Lee, D. Wedin, S. Naeem, and D. Bahauddin. 2001. Plant diversity enhances ecosystem responses to elevated $\mathrm{CO}_{2}$ and nitrogen deposition. Nature 410:809810.

Reich, P. B., D. Tilman, F. Isbell, K. Mueller, S. E. Hobbie, D. F. B. Flynn, and N. Eisenhauer. 2012. Impacts of biodiversity loss escalate through time as redundancy fades. Science 336:589-592.

Ricklefs, R. E. 1977. Environmental heterogeneity and plant species diversity: a hypothesis. American Naturalist 111:376381.

Roscher, C., V. M. Temperton, M. Scherer-Lorenzen, M. Schmitz, J. Schumacher, B. Schmid, N. Buchmann, W. W.
Weisser, and E.-D. Schulze. 2005. Overyielding in experimental grassland communities - irrespective of species pool or spatial scale. Ecology Letters 8:419-429.

Schnitzer, S. A., and W. P. Carson. 2010. Lianas suppress tree regeneration and diversity in treefall gaps. Ecology Letters 13:849-857.

Schnitzer, S., J. Klironomos, J. HilleRisLambers, L. Kinkel, P. Reich, K. Xiao, M. Rillig, B. Sikes, R. Callaway, and S. Mangan. 2011. Soil microbes drive the classic plant diversityproductivity pattern. Ecology 92:296-303.

Tilman, D. 1977. Resource competition between plankton algae: an experimental and theoretical approach. Ecology 58: $338-348$.

Tilman, D. 1985. The resource-ratio hypothesis of plant succession. American Naturalist 125:827-852.

Tilman, D., and J. A. Downing. 1994. Biodiversity and stability in grasslands. Nature 367:363-365.

Tilman, D., P. Reich, J. Knops, D. Wedin, T. Mielke, and C. Lehman. 2001. Diversity and productivity in a long-term grassland experiment. Science 294:843-845.

Tilman, D., and D. Wedin. 1991. Plant traits and resource reduction for five grasses growing on a nitrogen gradient. Ecology 72:685-700.

Valladares, F., J. Zaragoza-Castells, D. Sanchez-Gomez, S. Matesanz, B. Alonso, A. Portsmuth, A. Delgado, and O. K. Atkin. 2008. Is shade beneficial for Mediterranean shrubs experiencing periods of extreme drought and late-winter frosts? Annals of Botany 102:923-933.

Van Auken, O. 2000. Shrub invasions of North American semiarid grasslands. Annual Review of Ecology and Systematics 31:197-215.

Van Ruijven, J., and F. Berendse. 2003. Positive effects of plant species diversity on productivity in the absence of legumes. Ecology Letters 6:170-175.

Wright, A., S. A. Schnitzer, I. A. Dickie, A. R. Gunderson, G. A. Pinter, S. A. Mangan, and P. B. Reich. 2013. Complex facilitation and competition in a temperate grassland: loss of plant diversity and elevated $\mathrm{CO}_{2}$ have divergent and opposite effects on oak establishment. Oecologia 171:449-458.

Zhang, Y., H. Y. H. Chen, and P. B. Reich. 2012. Forest productivity increases with evenness, species richness and trait variation: a global meta-analysis. Journal of Ecology 100:742-749.

\title{
Supplemental Material
}

\begin{abstract}
Appendix A
Allometric relationships for calculating pine seedling biomass (Ecological Archives E095-196-A1).
\end{abstract}

\section{Appendix B}

Further details about the two statistical models (Ecological Archives E095-196-A2).

\section{Appendix C}

The effects of herbaceous species richness and seedling size class on seedling relative growth rate (RGR) (Ecological Archives E095-196-A3).

\section{Appendix D}

The effects of species richness and size class on pine RGR at biweekly intervals (Ecological Archives E095-196-A4). 